\title{
Identification and assessment of kefir yeast potential for sugar/ethanol-resistance
}

\author{
M.G.C.P. Miguel, P.G. Cardoso, K.T. Magalhães-Guedes, R.F. Schwan \\ Departamento de Biologia, Universidde Federal de Lavras, Campus Universitário, Lavras, MG, Brazil.
}

Submitted: June 22, 2011; Approved: July 02, 2012.

\begin{abstract}
Biochemical and molecular analysis was used for identification of different kefir yeasts species from Brazil, Canada and the United States of America. The sugar/ethanol-resistant activity of the yeasts was evaluated. Saccharomyces cerevisiae and Kluyveromyces marxianus had the highest growth rates, suggesting biotechnological applications possible for these strains.
\end{abstract}

Key words: kefir, sugar/ethanol-resistant yeasts, yeasts diversity, rep-PCR, PCR-DGGE.

Kefir is a refreshing, naturally carbonated fermented dairy beverage with a slightly acidic taste, yeasty flavor and creamy texture (Miguel et al., 2010; Magalhães et al., 2011a, 2011b). Previous results have shown that two groups of microorganisms co-exist in milk kefir grains: lactic acid bacteria and yeast (Miguel et al., 2010; Magalhães et al., 2011a, 2011b). The presence of yeast enhances the organoleptic quality of the kefir beverage, promoting a strong and typically yeasty aroma, as well as a refreshing and pungent taste (Magalhães et al., 2011a, 2011b). Yeasts isolated from kefir could be used for research that aims to produce fermented alcoholic beverages and ethanol using other substrates, e.g. cheese whey. Cheese whey is the major by-product of the dairy industry, and its disposal without expensive sewage treatments represents a major source of environmental pollution. Some methods have been proposed for whey valorization by yeast (Dragone et al., 2009; Guimarães et al., 2010) including distilled beverages (Dragone et al., 2009) and kefir-like whey beverages (Paraskevopoulou et al., 2003; Magalhães et al., 2010b, 2011a, 2011b). Seeking this possibility, the aim of this study was to isolate and identify kefir-derived yeast species from different countries (Brazil, Canada, and the United States of America) and to evaluate their potential for sugar/ethanol-resistance. The yeast diversity was also assayed using denaturing gradient gel electrophoresis (DGGE).

The samples of kefir grains were obtained from families (private households) that traditionally consumed the kefir beverage. These families were from locations in three different countries: Lavras (Brazil - BR), Bowmanville
(Canada - CA), and Fayette (United States of America USA). Each kefir grain sample was collected aseptically for phenotypic analysis (50 g of sample) and genotypic analysis (5 $\mathrm{g}$ of sample).

Yeasts were enumerated by plating $10 \mu$ Lof each diluted sample in triplicate using the surface spreading technique described by Magalhães et al. (2010a). Enumeration of yeasts was carried out using the culture media malt yeast glucose peptone extract (MYGP agar, Merck, Whitehouse Station, USA); $0.3 \%$ yeast extract, $0.3 \%$ malt extract, $0.5 \%$ peptone, $1.0 \%$ glucose, $2.0 \%$ agar) containing $100 \mathrm{mg} / \mathrm{L}$ chloramphenicol (Sigma, St. Louis, USA) and $50 \mathrm{mg} / \mathrm{L}$ chlortetracycline (Sigma, St. Louis, USA) to inhibit bacterial growth. After spreading, plates were incubated at $28^{\circ} \mathrm{C}$ for $120 \mathrm{~h}$ and colony forming units $\left(\log _{10} \mathrm{cfu} / \mathrm{g}\right)$ were quantified. Colonies were taken at random for identification from each plate containing isolated colonies (the number of colonies used for identification was equal to the square root of the total number of colonies on that plate). The phenotypic characteristics of all yeast isolates were determined by their morphology and spore formation, as well as their assimilation and fermentation of different carbon sources (Magalhães et al., 2010a). For the selection of yeast isolates, molecular biology-based grouping of the isolates was performed using repetitive extragenic palindromic (repPCR) using the primer (GTG) ${ }^{5}$ (5'-GTG GTG GTG GTG GTG-3'). The screening was performed with all 125 isolates. The cluster was done only by the different profiles obtained from rep-PCR technique. DNA was extracted from the pure cultures as described by Pereira et al. (2010). The 
yeast Saccharomyces cerevisiae UFLA GF33 was used as the reference strain. The rep-PCR profiles were normalized, and cluster analysis was performed using Bionumerics 2.50 (Applied Maths, Sint-Martens-Latem, Belgium). After the selected isolates by rep-PCR technique were identified by Internal Transcribed Spacer (ITS) region analysis (Magalhães et al., 2011c).

In addition, a culture-independent method was used to analyze each sample. Kefir grains ( $5 \mathrm{~g}$ ) from the different countries were transferred into a plastic tube and subjected to DNA extraction using a NucleoSpin Tissue kit (Macherey-Nagel, Düren, Germany) according to the manufacturer's instructions. Genomic DNA was used as a template for PCR amplification of yeast ribosomal target regions, and the products from the PCR reaction were then analyzed by PCR-DGGE). The primers NS3fGC and YM951r were used for partial ribosomal 18S DNA amplification as described by Magalhães et al. (2010a). Selected bands from the PCR-DGGE gels were excised and reamplified using the same primers without GC clamp. The sequencing of portions of the ITS region from the yeast isolates and $18 \mathrm{~S}$ rRNA gene amplicons were performed by the Central Laboratory of Molecular Biology - LCBM/UFLA (Lavras, MG, Brazil). Sequence similarity was analyzed using the BLAST database from GenBank (http://www.ncbi.nlm.nih.gov/BLAST/).

One representative microorganism from each identified microbial species and each kefir grain was inoculated in triplicate in $2.0 \mathrm{~mL}$ culture medium MYGP medium containing $100 \mathrm{mg} / \mathrm{L}$ chloramphenicol and $50 \mathrm{mg} / \mathrm{L}$ chlortetracycline to inhibit bacterial growth. The medium was then supplemented with $0,4,6,9$ or $12 \% \mathrm{v} / \mathrm{v}$ ethanol (Merck, Whitehouse Station, USA), and the flasks were incubated at $28{ }^{\circ} \mathrm{C}$ for $120 \mathrm{~h}$. The ethanol resistance was determined by measuring biomass formation. The yeast Saccharomyces cerevisiae UFLA GF33, which is ethanol-resistant and has a high growth rate under these conditions, was used as a control. The ethanol-resistant yeasts were inoculated in triplicate in $2.0 \mathrm{~mL}$ of culture medium containing $10 \mathrm{~g} / \mathrm{L}$ yeast extract (Merck, Whitehouse Station, USA) and $20 \mathrm{~g} / \mathrm{L}$ peptone (Merck, Whitehouse Station, USA). This medium was supplemented with 100 or 250 g/L (Osho, 2005; Miguel et al., 2010) glucose (Merck, Whitehouse Station, USA) or lactose (Merck, Whitehouse Station, USA). Sugar resistance was determined as described above.

The enumeration values $(\mathrm{cfu} / \mathrm{g}$ ) of the isolated viable yeasts ranged from a minimum value of $7.43 \pm 0.01 \log _{10}$ $\mathrm{cfu} / \mathrm{g}$ in the sample from Canada to a maximum value of $9.57 \pm 0.03 \log _{10} \mathrm{cfu} / \mathrm{g}$ in the sample from Brazil, with the sample from the USA falling in between at $8.38 \pm 0.02 \log _{10}$ $\mathrm{cfu} / \mathrm{g}$. A total of 125 isolates were obtained from kefir grain samples and grouped for molecular characterization using the $(\mathrm{GTG})^{5}$-PCR technique (Figure 1a). The cluster was grouped by Bionumerics 2.50 software (Applied Maths,
Sint-Martens-Latem, Belgium). Cluster analysis using $(\mathrm{GTG})^{5}$-PCR patterns resulted in the identification of two groups referred to as Y1 and Y2 at the level of $45 \%$ similarity. The Y1 group was subdivided into three subgroups (Y1A, Y1A1 and Y1B) with greater than 70\% similarity. The Y1A group consists of isolates of the Saccharomyces genus. The Y1A1 group is composed of Saccharomyces cerevisiae isolated, which are grouped at the level of $\sim 96 \%$ similarity. This group therefore is grouped with Saccharomyces bayanus strain, forming the group Y1A to $\sim 79 \%$ similarity. These isolates were derived from kefir grains from Brazil, Canada and the USA. Group Y2 contained 14 isolates that demonstrated less similarity with UFLA GF33. These isolates were also present in kefir grains from Brazil, Canada and the USA. The high incidence of band differences between yeast isolates makes the rep-PCR technique efficient for distinguishing between different strains. Isolates representing different branches in the $(\mathrm{GTG})^{5}$-PCR fingerprinting analysis were chosen for partial sequencing of the PCR-amplified ITS region.

The 125 yeasts isolated were identified by phenotypic and genotypic techniques as Saccharomyces cerevisiae (11 from Brazil, 7 from Canada and 9 from the USA), Saccharomyces sp (3 from Brazil, 3 from Canada and 5 from the USA), Saccharomyces bayanus (2 from Brazil), Zygosaccharomyces mellis (3 from Brazil), Dekkera anomala (3 from Brazil and 1 from Canada), Kluyveromyces marxianus (21 from Brazil, 16 from Canada and 19 from the USA), Pichia membranifaciens (1 from Brazil), Pichia guilliermondii (2 from Brazil and 1 from Canada), Pichia fermentans ( 5 from Brazil and 6 from the USA), Pichia anomala (1 from Canada and 1 from the USA), Candida parapsilosis (1 from Brazil and 1 from the USA) and Candida valdiviana (1 from Canada and 2 from the USA). The species Saccharomyces cerevisiae, Pichia fermentans, Saccharomyces bayanus, Kluyveromyces marxianus and Zygosaccharomyces mellis have been previously found in kefir grains (Magalhães et al., 2010a, 2011c; Miguel et al., 2011), whereas Dekkera anomala has not been reported as a microorganism present in kefir grains. Dekkera anomala, may have been introduced to the kefir grains as a contaminant during manipulation or transport. Another factor may be the species Dekkera anomala be part of the original microbiota of these grains, because different kefir grains may have diverse microbiota (Miguel et al., 2010, 2011; Magalhães et al., 2011c). This species was identified in kefir grain samples from Brazil and Canada. Saccharomyces spp. were also identified in this study. Sequencing of the ITS region confirmed that the isolates are closely related to Saccharomyces cerevisiae and Saccharomyces sp., with 99.2-100\% similarity. However, $S$. cerevisiae, $S$. bayanus and $S$. pastorianus are very closely related, so to obtain an unambiguous identification, the isolates were differentiated using species-specific PCR primer pairs homologous to the $\mathrm{HO}$ genes of $S$. cerevisiae 

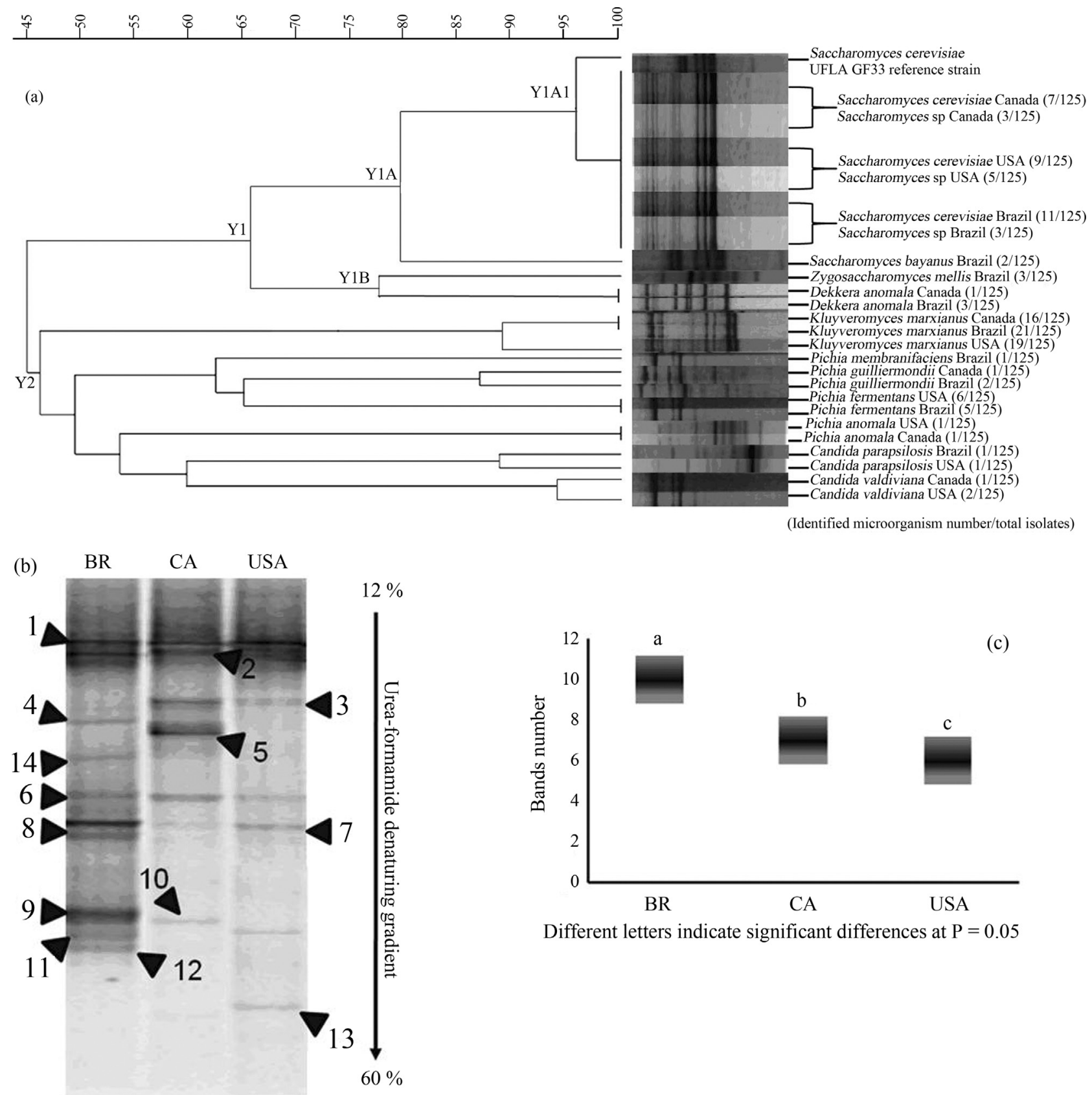

Different letters indicate significant differences at $\mathrm{P}=0.05$

Figure 1 - Molecular analysis from kefir grains samples of different countries. (a) Cluster analysis of the profiles obtained by rep-PCR of the yeasts isolated from kefir grains. (b) DGGE profiles of yeast $18 \mathrm{~S}$ rDNA fragments amplified from kefir grains samples of different countries. Abbreviations: BR Brazil; CA - Canada; USA - United States of America. Band 1 - Pichia fermentans FJ770542.1 Band 2 - Pichia guilliermondii EF197951.1 Bands 3 Candida valdiviana FN652300.1 Band 4 and 8 - Zygosaccharomyces sp. AF17728.1 Band 5 - Lachancea meyersii AY645661.1 Band 6 - Saccharomyces cerevisiae EU649673.1 Band 7 - Kluyveromyces marxianus GM620595 Band 9 - Saccharomyces bayanus D89889.1 Band 11 - Candida sp. GI190714325 Band 14 - Kazachatania unispora EU789404.1 Band 10, 12, 13 - not identified. (c) Band numbers of DGGE profiles of yeast communities from kefir grains statistically compared by Duncan's multiple range test using the SPSS version 10.0. $\mathrm{p}=0.05$.

(Pereira et al., 2010). A single amplicon of approximately 40 bpconfirmed the identity of the Saccharomyces spp. as S. cerevisiae (data not shown).

In addition to the culture-based methods, the yeast communities from the kefir samples were investigated using DGGE analysis of the PCR-amplified 18S rRNA gene. Kefir grain samples from Brazil had the greatest band di- versity according to a statistical comparison using Duncan's multiple range test $(\mathrm{p}=0.05$; Figure $1 \mathrm{c})$, suggesting that this sample had the greatest diversity of yeast species. The CA and USA grain samples showed no significant differences in band diversity. To determine the composition of the yeast communities from the kefir samples, the bands were excised from the DGGE gel, and the DNA was 
isolated and sequenced. Figure $1 \mathrm{~b}$ shows the DGGE profiles of yeast 18S rDNA fragments amplified from kefir grains samples of different countries. The bands were identified based on a search for similar sequences in GenBank as follows: band 1 - Pichia fermentans, band 2 - Pichia guilliermondii, band 3 - Candida valdiviana, bands 4 and 8 - Zygosaccharomyces sp., band 5 - Lachancea meyersii, band 6 - Saccharomyces cerevisiae, band 7 Kluyveromyces marxianus, band 9 - Saccharomyces bayanus and band 14 - Kazachatania unispora. Despite the fact that the Zygosaccharomyces sp. and Candida sp. could be identified only at the genus level, Zygosaccharomyces fermentati and Candida valdiviana or Candida parapsilosis were the most probable species because they were sporadically isolated from kefir grains in this study. The Zygosaccharomyces sp. (bands 4 and 8) appeared in the DGGE gel from the Brazil sample in two different positions. The multiple banding patterns may be due to sequence heterogeneity between multiple copies of the $18 \mathrm{~S}$
rDNA in this strain (Miguel et al., 2011). Lachancea meyersii and Kazachatania unispora were not isolated by culture-dependent methods, but had been previously identified in kefir grains by Magalhães et al. (2010a, 2010b). It was not possible to identify band 10,12 and 13; the bands were excised from the gel, but DNA could not be recovered for sequencing.

Twenty-five kefir yeasts (grouped by rep-PCR) isolated from fermented milk were screened for ethanol and sugar (glucose/lactose) tolerance (Figure 2). Only the isolates of Saccharomyces cerevisiae, Saccharomyces sp. and Kluyveromyces marxianus were able to grow at a concentration of $12 \%(\mathrm{v} / \mathrm{v})$ ethanol. Figure 2 shows the identities of the isolates as well as the percentage of ethanol (v/v) that inhibited the growth of the other isolates. All of the ethanol-tolerant yeasts were tested in different concentrations of both glucose and lactose, and all reached their maximum growth rates at a sugar concentration of 25\% (v/v) (Figure 2). Saccharomyces cerevisiae and Kluyveromyces

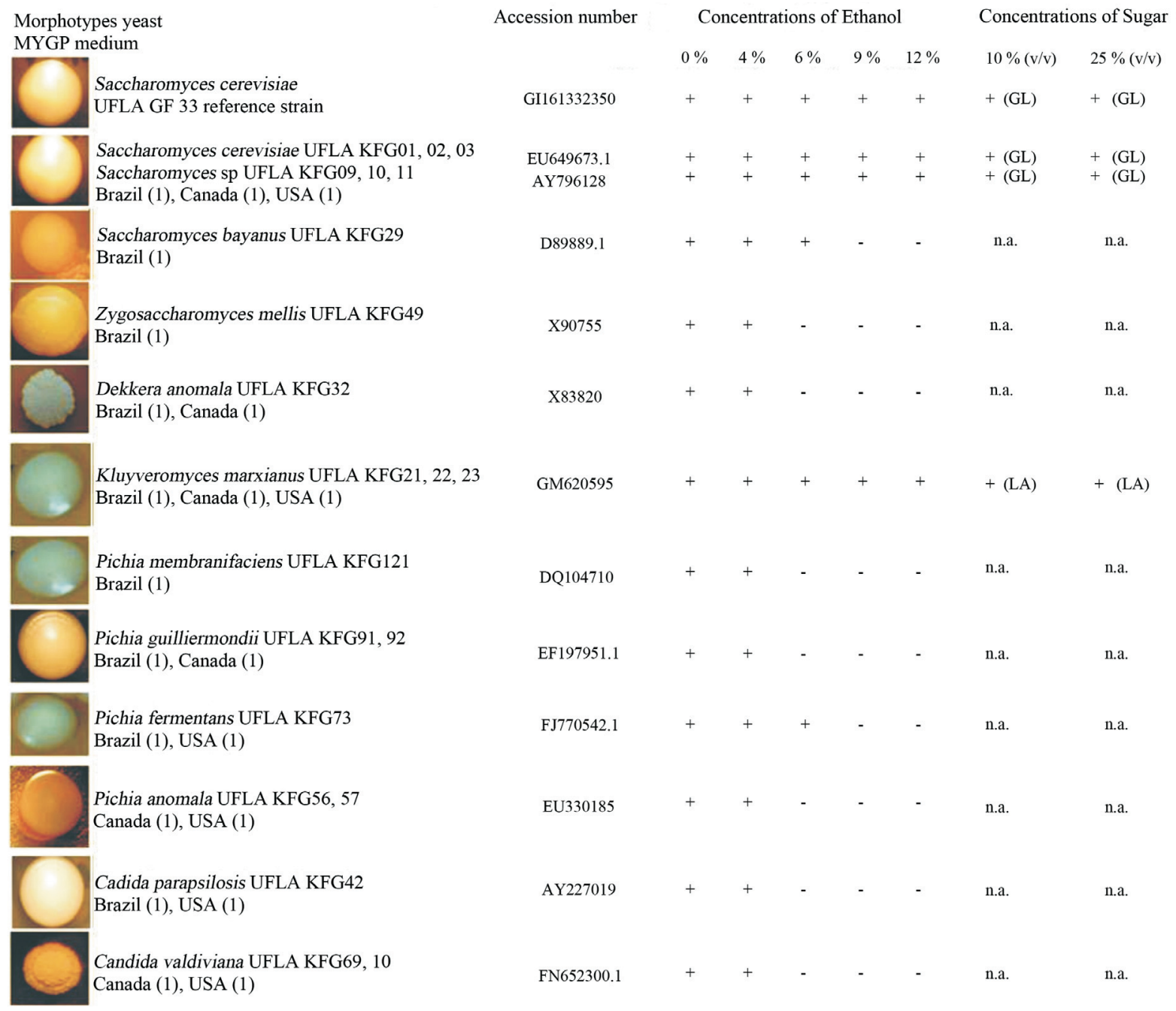

Figure 2 - Screening sugar/ethanol-tolerance kefir yeasts. (+) biomass formation; (-) biomass not formation. Abbreviations: (GL) Glucose; (LA) Lactose; n.a. not analyzed. The values in parentheses refer to the yeast isolates number tested of each country. 
marxianus showed the highest growth rates. The fact that the yeast strains were able to grow in media containing relatively high concentrations of sugar demonstrates the ability of the 7 ethanol-tolerant yeast strains to withstand osmotic stress. This observation is in agreement with the suggestion of Osho (2005), who stated that ethanol-tolerant yeasts tend to be sugar-tolerant. Ekunsanmi and Odunfa (1990) asserted that the combination of sugar tolerance and alcohol tolerance is an advantage when a yeast species is being considered for industrial use, especially where ethanol is being produced. Osho (2005) pointed out that ethanol tolerance is particularly important because high concentrations of ethanol in the medium are difficult to avoid during fermentation, while substrate inhibition can be avoided through step-wise addition of substrate. In medium where wine is the ultimate product, sugar tolerance by the wine yeast strains allows a larger initial amount of sugar to be used. The isolation of highly ethanol- and sugar-tolerant yeast strains from kefir grains will allow further investigation into the practical applications of the isolated strains.

Cheese whey is the main by-product of the dairy industry and has been increasingly recognized as a source of many bioactive valuable compounds. However, the most abundant component in whey is lactose (ca. 5\% w/v) (Paraskevopoulou et al., 2003; Pescuma et al., 2008; Dragone et al., 2009; Guimarães et al., 2010; Magalhães et al., 2010b, 2011a, 2011b). Due to the large lactose surplus generated, its conversion to bio-ethanol has long been considered as a possible solution for cheese whey bioremediation. Because of this, emphasis has been given to advances in engineering a consortium of $K$. marxianus and S. cerevisiae strains for efficient cheese whey-to-ethanol bioprocesses (Dragone et al., 2009; Guimarães et al., 2010). The yeasts isolated in this study (particularly those with high ethanol- and sugar-tolerance, i.e., K. marxianus and S. cerevisiae) suggest biotechnological applications possible for these strains e.g. represent new avenues for the study of the alcoholic fermentation of cheese whey. Another suggestion, the production of fermented alcoholic beverages produced upon whey fermentation by a consortium of $K$. marxianus and $S$. cerevisiae strains (identified and analyzed in this study) could be an interesting alternative for cheese whey utilization. Cheese whey fermentation by these yeasts could decrease the high lactose content in cheese whey, producing mainly lactic acid and other metabolites such as aroma compounds contributing to the flavor and texture and increasing carbohydrate solubility and sweetness of the end product (Pescuma et al., 2008). Manufacture of beverages through lactic fermentations can provide desirable sensory profiles and have already been considered an option to add value to cheese whey (Pescuma et al., 2008).

\section{Acknowledgments}

The authors acknowledge to those who provide the kefir grains samples for use in this study and Coordenação de Aperfeiçoamento de Pessoal de Nível Superior (CAPES), Conselho Nacional de Desenvolvimento Científico e Tecnológico (CNPq) and Fundação de Amparo à pesquisa do Estado de Minas Gerais (FAPEMIG) for financial support.

\section{References}

Dragone G, Mussatto SI, Oliveira JM, Teixeira JA (2009) Characterisation of volatile compounds in an alcoholic beverage produced by whey fermentation. Food Chem 112:929935.

Ekunsanmi TJ, Odunfa SA (1990) Ethanol tolerance, sugar tolerance and invertase activities of some yeasts strains isolated from steep water of fermenting cassava tubers. J Appl Bact 69:672-675.

Guimarães PMR, Teixeira JA, Domingues L (2010) Fermentation of lactose to bio-ethanol by yeasts as part of integrated solutions for the valorization of cheese whey. Biotechnol Adv 28:375-384.

Magalhães KT, Dias DR, Pereira GVM, Oliveira JM, Domingues L, Teixeira JA, Silva JBA, Schwan RF (2011a) Chemical composition and sensory analysis of cheese whey-based beverages using kefir grains as starter culture. Int J Food Sci Tech 46:871-878.

Magalhães KT, Dragone G, Pereira GVM, Oliveira JM, Domingues L, Teixeira JA, Silva JBA, Schwan RF (2011b) Comparative study of the biochemical changes and volatile compounds during the production of novel whey-based kefir beverages and traditional milk kefir. Food Chem 126:249253.

Magalhães KT, Pereira GVM, Campos CR, Dragone G, Schwan RF (2011c) Brazilian kefir: Structure, microbial communities and chemical composition. Braz J Microbiol 42:693702.

Magalhães KT, Pereira GVM, Dias DR, Schwan RF (2010a) Microbial communities and chemical changes during fermentation of sugary Brazilian kefir. World J Microbiol Biotechnol 33:1-10.

Magalhães K, Pereira MA, Nicolau A, Dragone G, Domingues L, Teixeira JA, de Almeida Silva JB, Schwan RF (2010b) Production of fermented cheese whey-based beverage using kefir grains as starter culture: Evaluation of morphological and microbial variations. Biores Tech 101:8843-8850.

Miguel MGCP, Cardoso PG, Lago LA, Schwan RF (2010) Diversity of bacteria present in milk kefir grains using culturedependent and culture-independent methods. Food Res Inter 43:1523-1528.

Miguel MGCP, Cardoso PG, Magalhães KT, Schwan RF (2011) Profile of microbial communities present in tibico (sugary kefir) grains from different Brazilian States. World J Microbiol Biotechnol 27:1875-1884.

Osho A (2005) Ethanol and sugar tolerance of wine yeasts isolated from fermenting cashew apple juice. African J Biotechnol 4:660-662.

Paraskevopoulou A, Athanasiadis A, Blekas IG, Koutinas AA, Kanellaki M, Kiosseoglou V (2003) Influence of polysaccharide addition on stability of a cheese whey kefir-milk mixture. Food Hydroc 17:615-620.

Pereira GVM, Ramos CL, Galvão C, Dias ES, Schwan RF (2010) Use of specific PCR primers to identify three important in- 
dustrial species of Saccharomyces genus: Saccharomyces cerevisiae, Saccharomyces bayanus and Saccharomyces pastorianus. Lett Appl Microbiol 51:131-137.

Pescuma M, Heberta EM, Mozzia F, Valdez GF (2008) Whey fermentation by thermophilic lactic acid bacteria: Evolution of carbohydrates and protein content. Food Microbiol 25:442-451.

All the content of the journal, except where otherwise noted, is licensed under a Creative Commons License CC BY-NC. 